\title{
SOME MATHEMATICAL PROBLEMS IN BIOLOGY
}

\author{
BY S. I. RUBINOW
}

\begin{abstract}
A survey is presented of some mathematical problems encountered in biological studies. A brief description of the problems to be discussed are as follows. 1. Blood flow: The mean flow of blood through arteries and veins does not behave in accordance with Poiseuille's law all the time. 2. Tracer analysis: The inferences to be drawn from radioactive tracer studies of physiological systems, called compartment analysis, is an inverse problem. 3. Cell populations: The growth of cell populations is conveniently described by differential equations which utilize either age and time or maturity and time as independent variables.
\end{abstract}

I would like to take this opportunity to tell you about some of the mathematical problems I have encountered in studying biological phenomena during the past ten years or so. These phenomena are all describable by differential equations. However, I think you will see that the problems with which they are associated have novel features when compared to those usually encountered in engineering and the physical sciences. My descriptions will be unsatisfactory in the sense that they will be very brief. However, brevity will permit me to present you with an overview of several biomathematical problems deriving from biological experience.

Steady blood flow. I will describe first a problem in biomechanics, because the latter is one area of biology in which the underlying mathematical theory is very well understood. When the mean steady volume flux of blood through the vena cava of a dog is measured as a function of the pressure difference between the upstream end and the downstream end, a curve is determined [2], as illustrated in Figure 1. Similar observations have been made of viscous fluid flow through a rubber tube [2], as shown in Figure 2. In fact, the observed blood flux through the entire circulatory system of the dog displays similar properties [2]-[5]. In determining the curves of Figures 1 and 2, the upstream pressure was held fixed while the downstream pressure was decreased. The noteworthy feature of these experiments is that when the pressure difference increases beyond a certain value, the flux does not increase, unlike the flow through a rigid tube.

A simple theory of viscous fluid flow through a tube can account for these observations provided the collapsibility of the tube is accounted for [6]. In the

This article is the text of an address presented to the American Mathematical Society at its meeting in New York on April 10-13, 1974. It is based on some of the material presented in the author's monograph [1]; received by the editors October 16, 1974.

AMS (MOS) subject classifications (1970). Primary 92A05.

Key words and phrases. Mathematical biology, blood flow, tracer analysis, compartment systems, cell population growth, cell density function. 


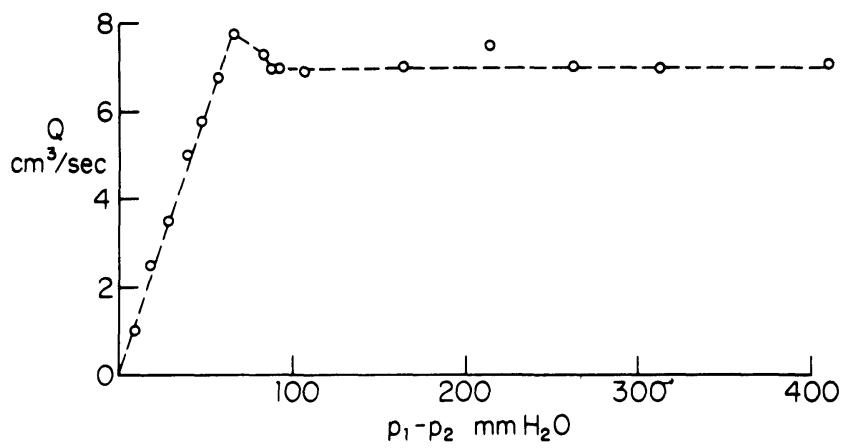

FIGURE 1. The steady flux of blood through the superior vena cava of a dog is shown as a function of the pressure difference $p_{1}-p_{2}$. Here $p_{1}$ is the pressure in the jugular vein (upstream), held fixed, and $p_{2}$ is the downstream pressure which was varied. From Rubinow and Keller [6], redrawn from the work of Brecher [2]. ${ }^{1}$

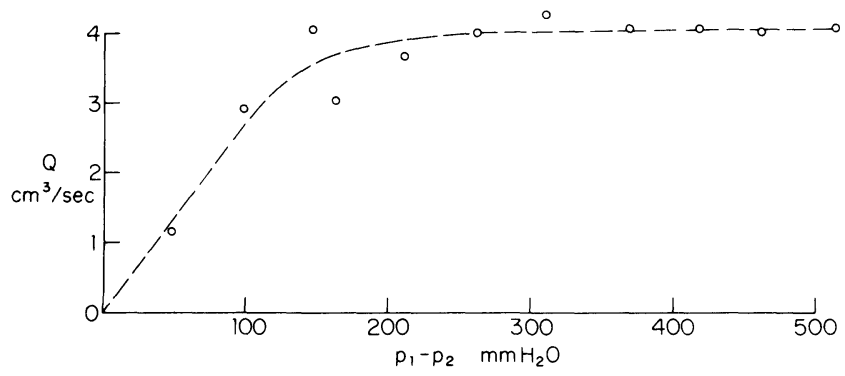

FIGURE 2. The steady viscous fluid flux through a collapsible rubber tube is shown as a function of the outlet pressure $p_{2}$ for fixed inlet pressure $p_{1}$. From Rubinow and Keller [6], redrawn from the work of Brecher [2].

interest of simplicity, I will present the theory for a tube of circular cross section only. Thus, we assume an incompressible fluid of viscosity $\mu$ is flowing steadily through an elastic tube of length $L$. Let the upstream and downstream pressures be $p_{1}$ and $p_{2}$, respectively, while the pressure surrounding the tube is $p_{0}$. At a given distance $z$ along the tube, the pressure is denoted by $p(z)$ (see Figure 3). The cross sectional area of the tube is assumed to depend only on the transmural pressure difference $p(z)-p_{0}$. In addition, we assume that Poiseuille's law holds locally, so that the steady flux $Q$ is given as

$$
Q=-\left(\pi a^{4} / 8 \mu\right)(d p / d z) .
$$

Here $a$ is the radius of the tube. However, the radius $a$ depends on the pressure $p$, for example, in accordance with the law of Laplace. Assuming the tension $T(a)$ in the tube wall is known as a function of the radius, it follows that the function $a=a\left[p(z)-p_{0}\right]$ is determined by the condition of equilibrium

$$
T(a) / a=p(z)-p_{0} .
$$

${ }^{1}$ Figures 1, 4, 5, 6, 10 and 11 are published from Mathematical problems in the biological sciences with permission. Copyright 1973 by Society for Industrial and Applied Mathematics. 


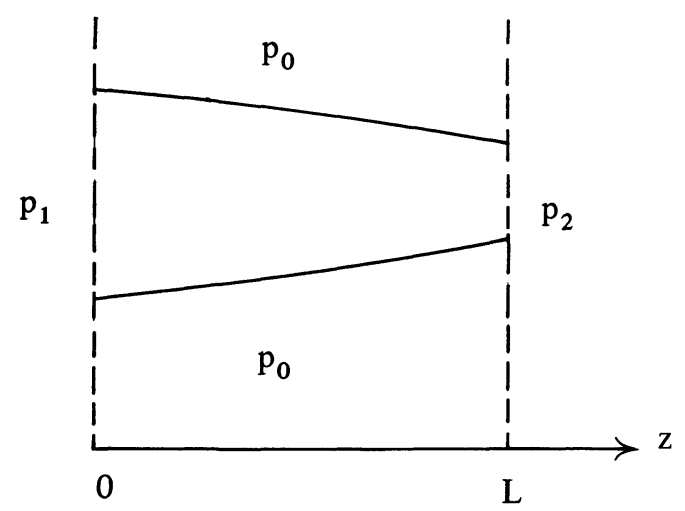

FIGURE 3. Schematic diagram of viscous fluid flow through an elastic tube of circular cross section, with upstream pressure $p_{1}$, downstream pressure $p_{2}$, and external pressure $p_{0}$.

Integrating equation (1) between the limits 0 and $L$, we readily obtain the result

$$
Q L=\frac{\pi}{8 \mu} \int_{p_{2}-p_{0}}^{p_{1}-p_{0}}\left[a\left(p^{\prime}\right)\right]^{4} d p^{\prime} .
$$

This equation is the principal result of the theory. It tells us that $Q$ is proportional to $L^{-1}$, and that it depends on the two pressure differences, $p_{1}-p_{0}$ and $p_{2}-p_{0}$. When $p_{1}-p_{2}$ is small, the mean value theorem indicates that $Q$ is proportional to $p_{1}-p_{2}$, just as for a rigid tube, and in agreement with the initial rising portion of the flow curve of either Figure 1 or Figure 2. However, when $p_{2}-p_{0}$ is large and negative, $Q$ approaches a limit if $p_{1}-p_{0}$ remains fixed, assuming the integral exists. The latter condition will be satisfied if the tube cross section decreases rapidly enough as the pressure difference $p_{2}-p_{0}$ decreases.

We applied this theory to the flow through an artery. Physiologists have determined tension-radius curves for human arteries, and they have the general shape shown in Figure 4 [7]. Here $a=1$ represents the resting radius of the artery when the transmural pressure difference is zero. We assume that the curve is extended in sigmoidal fashion for $a<1$, in agreement with the known properties of rubber tubes, the trachea and the small and medium bronchi [8].

Figure 5 shows the calculated quantity $Q L$ as a function of $p_{2}-p_{0}$, for various values of the difference between the inlet pressure $p_{1}$ and the external pressure $p_{0}$. These curves are in qualitative agreement with those shown in Figures 1 and 2.

When the external pressure is sufficiently great, buckling occurs, and the tube cross section is no longer circular, as we all know from trying to drink ice cream sodas through a straw too quickly. The buckling of elastic tubes is illustrated in Figure 6 by observations of the cross sections of rubber tubing in various stages of collapse during flow [9]. The theory presented must be modified by replacing the coefficient $\left(\pi a^{4} / 8 \mu\right)$ by a suitable generalized 


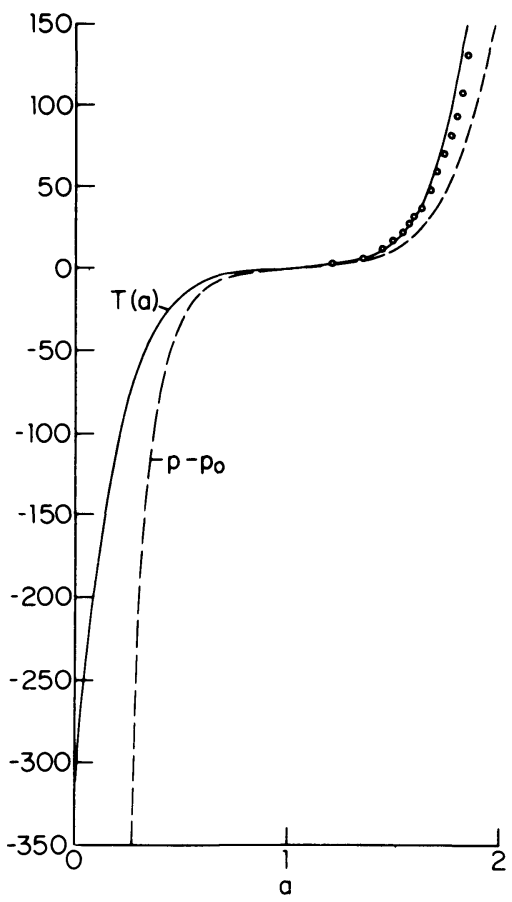

FIGURE 4. The solid line is a tension-length diagram for the external iliac artery, based on the experimental observations (circles) of Roach and Burton [7]. The dashed line represents $p-p_{0}$ as given by equation (2). From Rubinow and Keller [6].

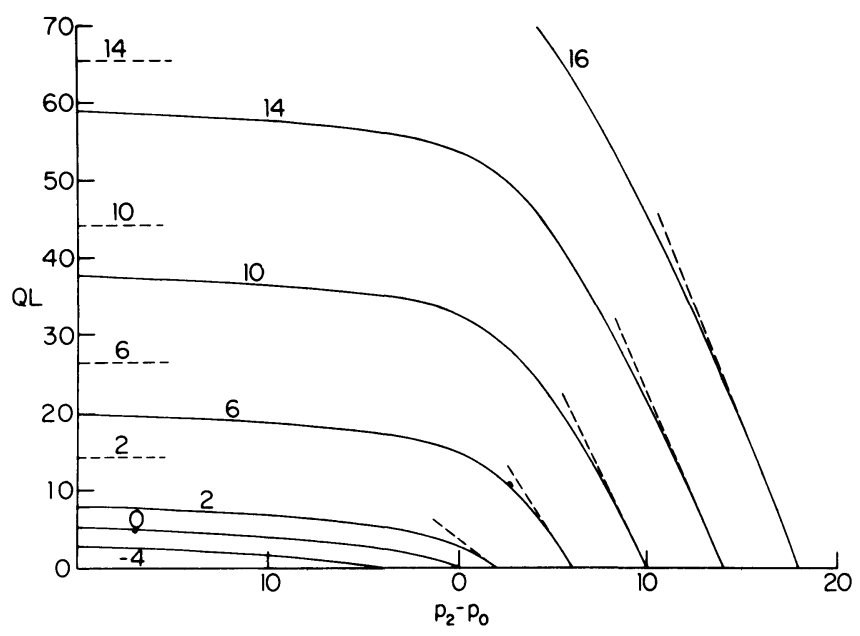

FIGURE 5. Theoretical curves of $Q L$ as a function of $p_{2}-p_{0}$ for various fixed values of the pressure difference $p_{1}-p_{0}$. The dashed horizontal lines represent asymptotes for which $p_{2}-p_{0} \rightarrow-\infty$. From Rubinow and Keller [6]. 
conductivity $\sigma$. The slope of the cross section of such a collapsed tube can be calculated from elasticity theory [10], and the conductivity $\sigma$ can then be found by solving a suitable flow problem. The calculated shapes of the buckled cross section of such a tube are very similar to those observed in rubber tubes. The qualitative features of the theory presented for the flow through a collapsible tube are not thereby affected.

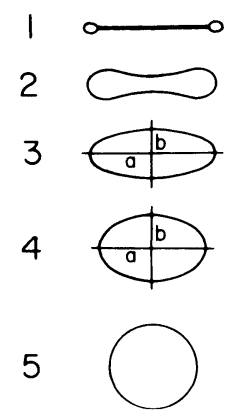

FIGURE 6. The cross sections of Penrose rubber tubing in various stages of collapse during flow, as observed by Holt [9].

2. Tracer investigations of physiological systems. Physiological processes in multicellular organisms are often described as homeostatic, which means that the organism is maintaining the process at a steady state. An example of homeostasis is the maintenance in our blood of the level of glucose concentration. Perhaps the most ubiquitous method of investigating such processes utilizes radioactive tracers. These investigations lead to curves of radioactivity versus time, and their mathematical analysis is called compartmental analysis. I will present here the mathematical theory underlying such tracer studies [11].

By a compartment we mean a material (chemical species, biological entity) with a given size. Here size represents either mass or volume. The compartment possesses a steady state flux of matter into and/or out of it. A compartment system is a set of $n$ interconnected compartments. By interconnected we mean that there exists a steady state of flux between any two compartments. The flux may result from diffusion, chemical reaction, or other causes. The flux from compartment $i$ to compartment $j$ is usually different from the flux from $j$ to $i$. An example of a three-compartment system is the blood plasma, extracellular volume, and tissue cells, with respect to a given metabolite. Flux at a steady rate is permitted to or from the exterior.

Now assume that at time $t=0$, a pulse of labeled matter is introduced into a compartment. The labeled matter is small compared to the unlabeled matter in any of the compartments, and leaves undisturbed the steady state fluxes. Finally, it is assumed that the mass distribution in any compartment is homogeneous, so that labeled matter sampled from any compartment is 
representative of the matter in that compartment. The transient of labeled matter thereby introduced is designed to yield information about the steady state fluxes.

The compartment equations governing the labeled matter flux are assumed to be

$$
d \rho / d t=L \rho,
$$

where $\rho=\left\{\rho_{i}\right\}$ is a vector representing the labeled matter. Here $L$ is a matrix of order $n$, and $L_{i j}$ represents the steady state flux or fractional clearance rate from compartment $j$ to compartment $i, i \neq j$. The off-diagonal elements of $L$ are positive or zero, but the diagonal elements, which represent the total efflux rates from each compartment to the other compartments and the exterior, are all negative.

When compartments are observed, it is usually the concentration $x=\left\{x_{i}\right\}$ that is sampled as a function of time. Therefore, we shall rewrite the compartment equations by setting

$$
\rho=V x
$$

where $V$ is a diagonal matrix, $V_{i i}$ representing the (constant) compartment size of compartment $i$. Then

$$
d x / d t=V^{-1} L V x=M x .
$$

Equation (6) equally well represents the fundamental equations of compartmental systems. Of course the solution to this equation system is well known, but for our purposes, we shall write the solution in the following manner. Let the time-dependent vector $e$ be defined as

$$
e=\left\{e^{\lambda_{i}}\right\},
$$

where $\lambda_{i}$ are the eigenvalues of $L$, or equivalently, of $M$. It is assumed they are distinct. It can be shown that they must have negative or zero real parts [12]. In practice, I have never seen a biological example possessing complex roots. Then

$$
d e / d t=\Lambda e,
$$

where $\Lambda$ is a diagonal matrix, $\Lambda_{i i}=\lambda_{i}$. We write the solution to the compartmental equations as

$$
x=A e
$$

where $A$ is a coefficient matrix of order $n$. By substitution of equation (9) into equation (6), it follows that

$$
M A=A \Lambda
$$

In other words, the columns of $A$ are the eigenvectors of $M$.

In a given experiment, a component of $x$ is observed as a function of time, and by a curve-fitting procedure, fitted to a sum of exponentials, in accordance with (9). This fitting determines $n$, the order of the compartment 
system, $\Lambda$, and one row of the coefficient matrix $A$. Now we can state the problem of compartmental analysis, which you will recognize to be an inverse problem: Given, from observation, $\Lambda$ and partial knowledge of $A$, that is to say, one or more rows of $A$, we wish to determine the compatible matrices $L$ and $V$.

The problem can be epitomized by what I call the bathtub model of compartmental analysis: given a set of bathtubs, with a fixed level of water in each bathtub. Any pair of bathtubs is connected by two pipes, with steady and, in general, unequal flows from one bathtub to the other. Each bathtub has a faucet, turned on or off, and a drain, open or closed. The water fluxes through all the pipes maintain the water level in each bathtub constant. A known amount of dye is injected into one of the bathtubs at $t=0$. Each bathtub has a blender which homogenizes the dye concentration in it instantaneously. By observing the dye concentration as a function of time in one or more bathtubs, infer as much as you can about the fluxes through all the pipes, and the volume of water in each bathtub.

I will restrict my remarks here to inferences that can be made from observations of one compartment only. From (10), we can write down the following $n-1$ equations relating the matrix $M$ to experimentally determined quantities:

$$
M^{p} x(0)=A \Lambda^{p} e(0)=\left(d^{p} x / d t^{p}\right)_{t=0}, \quad p=1,2, \cdots, n-1 .
$$

In addition, we can write down the $n$ equations relating $M$ and its invariants:

$$
\begin{aligned}
& \sum_{i=1}^{n} M_{i i}=\sum_{j=1}^{n} \lambda_{i}, \\
& \sum_{i, j=1 ; i \neq j}^{n}\left(M_{i i} M_{j j}-M_{i j} M_{j i}\right)=\sum_{i, j=1 ; i \neq j}^{n} \lambda_{i} \lambda_{j}, \\
& \quad \cdot \\
& \quad \cdot \\
&|M|=\lambda_{1} \lambda_{2} \cdots \lambda_{n} .
\end{aligned}
$$

The above $(2 n-1)$ algebraic equations constitute an underdetermined set of equations for the $n^{2}$ elements of $M$. In making inferences regarding the manifold of those matrices $L$ and $V$ which are compatible with observation, we are usually aided by the knowledge that, because of biological considerations, certain matrix elements of $L$ must be null.

We have posed the following question: What are the minimal matrices of the system, those which permit the maximum number of null constraints that are compatible with observation? The answers to this and other such questions are easily visualized with the aid of graphs to represent the matrix $L$. For example, for $n=3$, if the same compartment is injected and observed, there are only four minimal graphs possible, as shown in Figure 7.

A node represents a compartment, and a branch represents an off-diagonal element of $L$. A directed line segment ending with an arrowhead represents 

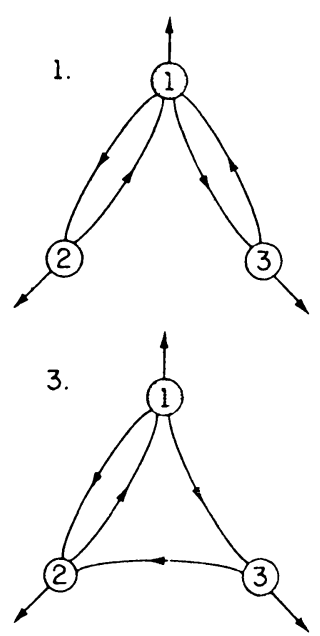
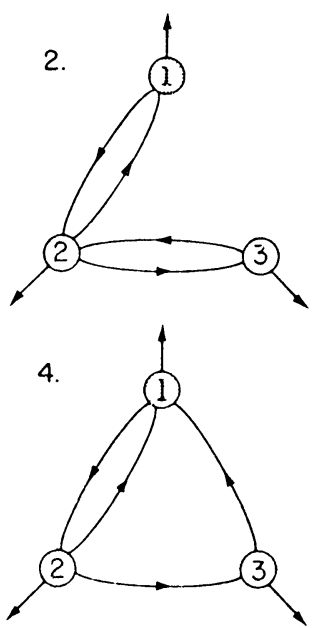

FIGURE 7. Graphs corresponding to the minimal matrices $L$ of a three-compartment system, for which compartment 1 is both injected and observed (shown shaded).

an excretion flux to the exterior. The diagonal element $L_{u}$ is represented by the sum of all directed line segments leaving the node $i$. In the graphs of Figure 7, additional null constraints can only be applied by setting at most two excretion rates equal to zero.

Finally, I will remark that there are special compartment systems for which it is possible to determine $L$ and $V$ uniquely, from the knowledge of $x_{1}(t)$ only [13]. An example of such a system is the closed catenary compartment system, which has the structure shown in Figure 8 . A catenary system is one for which the connection between compartments is "chainlike". A closed system is one for which all the excretion rates are zero. For a closed catenary system, $M$ has $3 n-2$ nonzero matrix elements, but only $2 n-2$ of them are unknown, because of the constraints imposed on the matrix $L$ by the closure property. They are uniquely determined from $x_{1}(t)$ by means of equations (11) and (12). Furthermore it can be shown that for this system,

$$
L=\tilde{M}
$$

where $\tilde{M}$ is the transpose of $M$. It is possible to make this inference by invoking the conservation of matter flow into and out of each compartment. In doing so, the possibility that a compartment is a source or a sink is excluded. Hence $L$ is likewise uniquely determined, and it also follows from the above relation that

$$
L=V \tilde{L} V^{-1}
$$

which determines the matrix $V$.

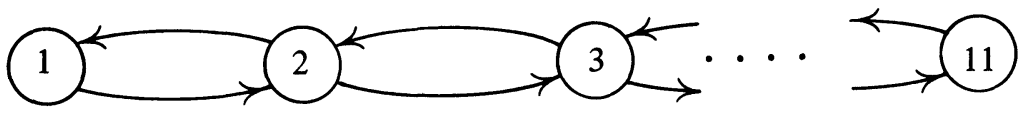

FIGURE 8. Graph for a closed catenary n-compartment system. 
3. Mathematical descriptions of cell population growth. The theoretical description of cell population growth has been greatly facilitated in recent years by the age-time formalism. Consider a population of living, proliferating cells. Each member has a chronological age $a$ assigned to it, the time elapsed since its birth. The population is described by a cell density function $n(a, t)$, where $n(a, t) d a$ is the number of cells in the age interval $a$ to $a+d a$, at time $t$. The total population at time $t, N(t)$, is obtained from it by summation,

$$
N(t)=\int_{0}^{\infty} n(a, t) d a .
$$

Even though the age of a cell is not usually an observable quantity, many cell properties are age dependent: cell division and the number of cells in mitosis, for example.

The cell density function is assumed to satisfy the equation [14], [15]

$$
\partial n / \partial t+\partial n / \partial a=-\lambda n, \quad a>0, t<0 .
$$

Usually $\lambda$ is a function of age only, $\lambda=\lambda(a)$, and represents the probability per unit time for cells in the age interval $a$ to $a+d a$ to disappear. Accordingly, the function $\lambda$ is divided into two parts,

$$
\lambda=\lambda_{m}+\lambda_{d},
$$

where the subscript $m$ stands for mitosis, and the subscript $d$ stands for death. The initial condition satisfied by $n$ is

$$
n(a, 0)=f(a) \text {, }
$$

where $f(a)$ is the given age distribution of the population at $t=0$. The boundary condition satisfied by $n$ is

$$
n(0, t)=2 \int_{0}^{\infty} \lambda_{m}\left(a^{\prime}\right) n\left(a^{\prime}, t\right) d a^{\prime},
$$

and represents the birth of two cells of age 0 from each cell of age $a^{\prime}$ that divides.

An alternative description of cell populations is the maturity-time representation [16] which introduces a cell density function $n(\mu, t)$, where $\mu$ is the maturation level or 'physiological age' of the cell. What do we mean by the latter term? Two possibilities are the cell volume, or the amounts of DNA in a cell. (In a simple bacterial cell such as E. coli, DNA synthesis proceeds in a linear fashion from the moment of birth.) The cell density function is assumed to satisfy the equation

$$
\partial n / \partial t+\partial(v n) / \partial \mu=-\lambda n, \quad \mu_{0}<\mu \leqq \mu_{1}, t>0 .
$$

Here $\mu_{0}$ and $\mu_{1}$ are the maturation levels of newborn cells and dividing cells, respectively, $v$ is the velocity of maturation, a prescribed function of $\mu$, and $\lambda$ represents the fractional time rate of cell disappearance due to death or other causes, but excluding cell division. The density function $n(\mu, t)$ satisfies 
an initial condition similar to (16),

$$
n(\mu, 0)=g(\mu),
$$

where $g(\mu)$ is the given maturation distribution of the population at $t=0$. The birth process is represented by the boundary condition

$$
n\left(\mu_{0}, t\right) v\left(\mu_{0}\right)=2 n\left(\mu_{1}, t\right) v\left(\mu_{1}\right),
$$

which states that the rate at which cells are born is twice the rate at which cells divide. A solution to the two equations can be given (but I will not do so here) in which the contribution to the population of successive generations is identified. Such a solution is useful for inferring the short time behavior of a population. These two mathematical theories lead to contrasting predictions concerning growing cell populations. I will illustrate this remark by only one example.

A fundamental property of cell populations, cultured under the ideal conditions of identical environment for each member, is that the generation time, the interval between cell birth and cell division, is not the same for each member of the population. The variability of the generation time appears to be an intrinsic reproducible property of a given cell strain. A good illustration of this property is provided in Figure 9, which shows the number of cells of a given age that divide, where all cells were considered to be of age zero at time zero. The cell population consisted of 766 cells of a single celled organism, Tetrahymena geleii [17].

In a related experiment, 50 cells, all at age zero, were cultured, and the population number carefully determined as a function of time. The results of this investigation could be used to test which of two contrasting hypotheses concerning the birth of these cells was valid. To wit, in the age-time formalism, it is assumed that a probabilistic rule governs cell division, so that there is no memory in a daughter cell of the parental generation time. An alternative hypothesis, in the framework of the maturity-time formalism, is that there is a heterogenous distribution of velocities of maturation, with some memory, from one generation to the next, of the generation time. The extreme form of this hypothesis is that each cell imparts to its daughters the same generation time that it possesses, that is to say, there is 'perfect memory' of the generation time.

With the first hypothesis, we can represent this experiment readily by the age-time formalism. The generation time distribution shown in Figure 9 determines the function $\lambda_{m}(a)$ in a straightforward manner. (In the quoted experiment, $\lambda_{d}$ is zero.) Then, with

$$
n(a, 0)=\mathrm{N}_{0} \delta(a)
$$

where $N_{0}$ is the total number of cells at age zero, and $\delta(a)$ is the Dirac delta function, we can determine $n(a, t)$ and hence $N(t)$.

The second hypothesis is formulated readily in the maturity-time formalism. Thus, we set $v=1 / T$, a constant, where $T$ is the generation time. Then the generation time distribution function shown in Figure 9 can be thought of 


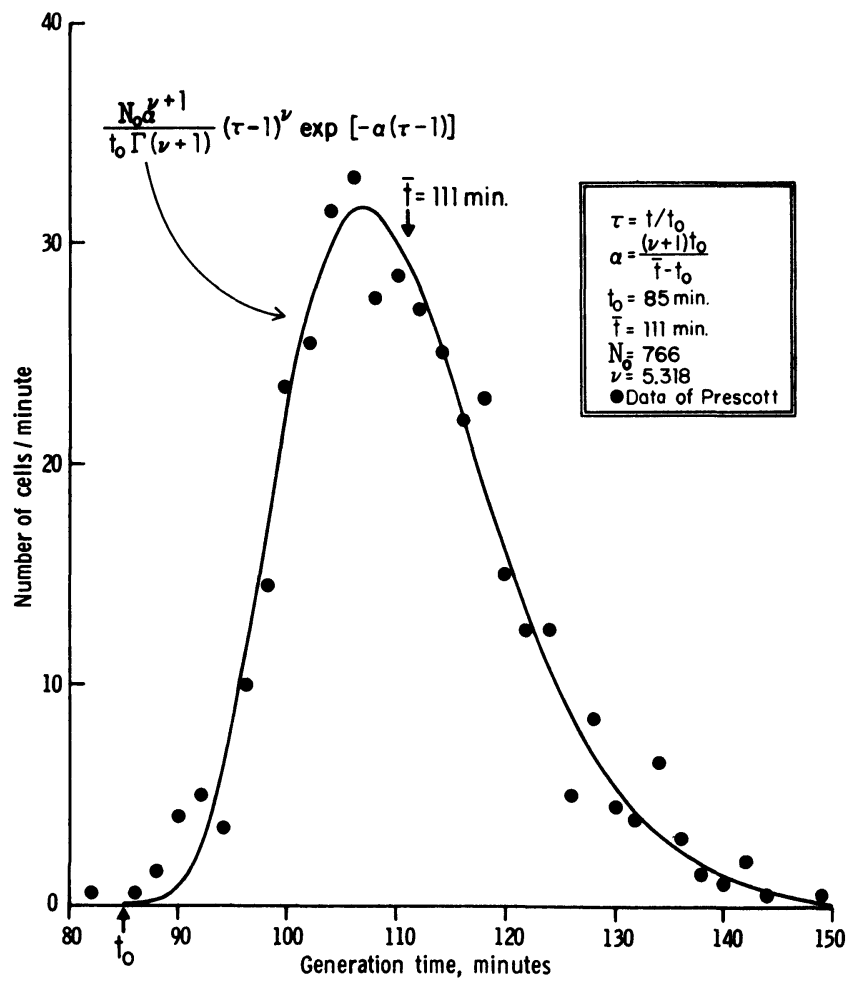

FIGURE 9. The data of Prescott [17] for the flux of cells of the HS strain of Tetrahymena geleii that divide, as a function of time. All cells are of age zero at time zero. The solid line is an arbitrary functional form, a gamma distribution, with parameter $\nu$ determined by the least square criterion. The distribution was otherwise constrained by the equations shown in the inset. From Rubinow [16].

as representing a distribution of subpopulations, each of generation time $T$, i.e.,

$$
n(\mu, t)=\int n_{T}(\mu, t) d T .
$$

The function $n_{\mathrm{T}}(\mu, t)$ is assumed to satisfy the maturity-time equation in the interval $0<\mu \leqq T$, subject to the conditions

$$
n_{T}(\mu, 0)=N_{0} u(T) \delta(\mu), \quad n_{T}(0, t)=2 n_{T}(T, t) .
$$

Here $u(T)$ is the generation time distribution of the initial cell population, given by the curve of Figure 9.

It should be obvious that this second model leads to a different prediction for the total population $N(t)$, than does the first model. It is true that $\mu$ could be replaced by the age $a$, and this model could then be considered as a special example of the age-time formalism. Conceptually, it is different, because there is no contribution to $\lambda$ from mitosis, and I prefer to consider it as a maturation-time model. 


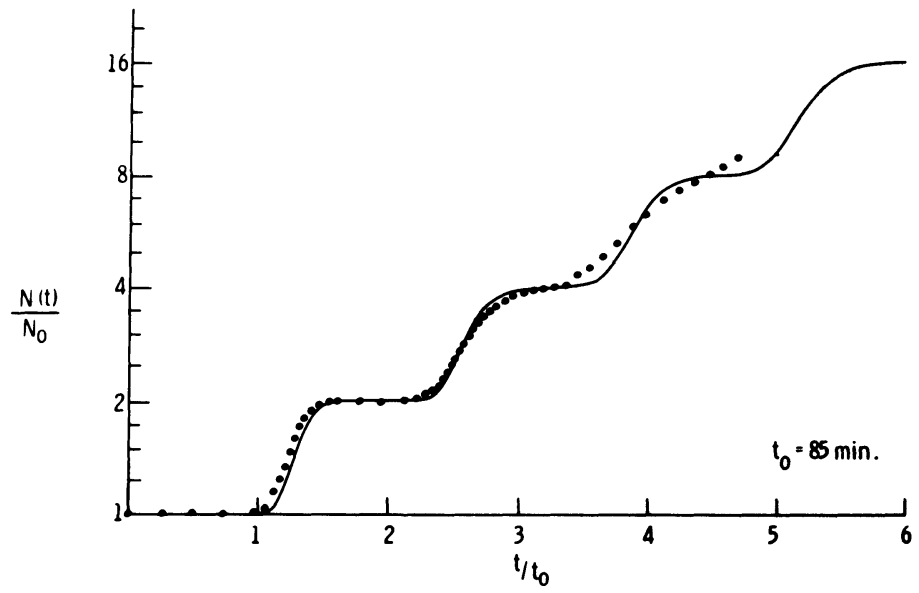

FIGURE 10. The circles represent the data of Prescott [17] for the population growth of an initially synchronized group of 50 cells of Tetrahymena geleii HS. A state of asynchronous growth appears rapidly because of generation time variability among the constituents of the population. The solid curve is the theoretically predicted growth curve for such a population in the age-time representation. From Rubinow [16].

The contrasting predictions of the 'probabilistic' and 'perfect memory' models, with the experimental observations of Prescott superimposed, are shown in Figures 10 and 11 respectively. It surprised me greatly that the 'perfect memory' model gave better agreement with observation. We can conclude from this agreement that, over a time span of several generations, cells do remember, on average, the generation time of their parents.

However, such a model cannot be correct for large times because, if it were, fast growing cells would ultimately swamp out the more slowly growing cells. The generation time distribution function of Figure 9 would then no longer be preserved in the 'wild' state. This objection can be resolved by introducing a

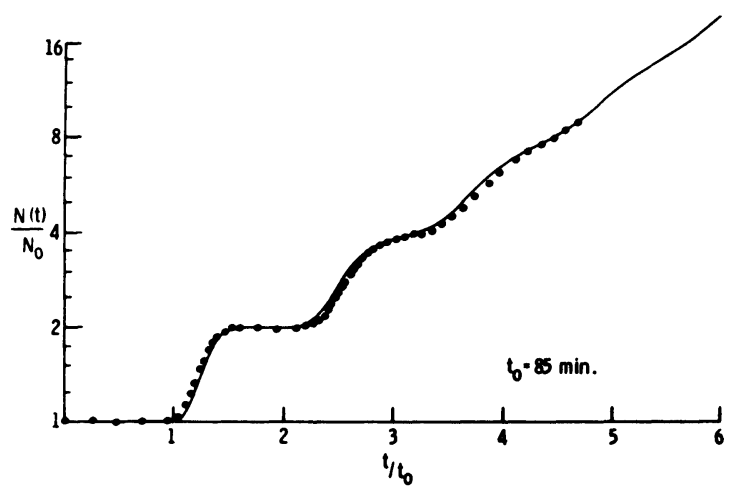

FIGURE 11. The solid line shows the total population as a function of time, that is theoretically predicted by the maturity-time representation. The data of Prescott are the same as shown in Figure 10. From Rubinow [16]. 
more complex theory containing three independent variables, age, time, and generation time [18]. However, the question as to how the distribution function $u(T)$ arises in the first instance, and maintains itself, remains an unresolved problem of intracellular dynamics.

\section{REFERENCES}

1. S. I. Rubinow, Mathematical problems in the biological sciences, Society for Industrial and Applied Mathematics, Philadelphia, 1973.

2. G. A. Brecher, Mechanism of venous flow under different degrees of aspiration, Amer. J. Physiol. 169 (1952), 423-433.

3. — Venous return, Grune \& Stratton, New York, 1956.

4. A. C. Guyton, Determination of cardiac output by equating venous return curves with cardiac response curves, Physiol. Rev. 35 (1955), 123-129.

5. — Venous return, Handbook of Physiology, Section 2: Circulation. Vol. II (W. F. Hamilton \& P. Dow, eds.), American Physiological Society, Washington, D.C., 1963, pp. 1099-1133.

6. S. I. Rubinow and J. B. Keller, Flow of a viscous fluid through an elastic tube with applications to blood flow, J. Theoret. Biol. 35 (1972), 299-313.

7. M. R. Roach and A. C. Burton, The reason for the shape of the distensibility curves of arteries, Canad. J. Biochem. \& Physiol. 35 (1957), 681-690.

8. D. L. Fry, A preliminary lung model for simulating the aerodynamics of the bronchial tree, Computers \& Biomed. Res. 2 (1968), 111-134.

9. J. P. Holt, Flow of liquids through "collapsible" tubes, Circ. Res. 7 (1959), 342-353.

10. J. E. Flaherty, J. B. Keller and S. I. Rubinow, Post buckling behavior of elastic tubes and rings with opposite sides in contact, SIAM J. Appl. Math. 23 (1972), 446-455.

11. S. I. Rubinow and A. Winzer, Compartment analysis: An inverse problem, Math. Biosciences 11 (1971), 203-247.

12. J. Z. Hearon, The kinetics of linear systems with special reference to periodic reactions, Bull. Math. Biophys. 15 (1953), 121-141. MR 14, 982.

13. S. I. Rubinow, On closed or almost closed compartment systems, Math. Biosciences 18 (1973), 245-253.

14. O. Scherbaum and G. Rasch, Cell size distribution and single cell growth in tetrahymena pyriformis GL, Acta Pathol. Microbiol. Scand. 41 (1957), 161-182.

15. H. Von Foerster, Some remarks on changing populations, The Kinetics of Cellular Proliferation (Frederick Stohlman, ed.), Grune \& Stratton, New York and London, 1959, pp. 382-407.

16. S. I. Rubinow, A maturity-time representation for cell populations, Biophys. J. 8 (1968), 1055-1073.

17. D. M. Prescott, Variations in the individual generation times of tetrahymena geleii HS, Exptl. Cell Res. 16 (1959), 279-284.

18. J. L. Lebowitz and S. I. Rubinow, $A$ theory for the age and generation time distribution of a microbial population, J. Math. Biol. 1 (1974), 17-36.

Current address: Graduate School of Medical Sciences, Cornell University, New York, New York 10021 